University of Wollongong

Research Online

Faculty of Engineering - Papers (Archive)

Faculty of Engineering and Information

Sciences

$1-1-2012$

\title{
Microstructural evolution during gaseous hydrogen charging of Zircaloy-4 processed by high-pressure torsion: A comparative study
}

\author{
Zhiyang Wang \\ University of Wollongong, zw603@uow.edu.au \\ Huijun Li \\ University of Wollongong, huijun@uow.edu.au \\ Ulf Garbe \\ The Bragg Institute, New Illawarra Road, Lucas Heights, NSW, Australia \\ Mark D. Callaghan \\ University of Wollongong, mcallagh@uow.edu.au \\ Yanbo Wang \\ University of Sydney
}

See next page for additional authors

Follow this and additional works at: https://ro.uow.edu.au/engpapers

Part of the Engineering Commons

https://ro.uow.edu.au/engpapers/4878

\section{Recommended Citation}

Wang, Zhiyang; Li, Huijun; Garbe, Ulf; Callaghan, Mark D.; Wang, Yanbo; and Liao, Xiaozhou: Microstructural evolution during gaseous hydrogen charging of Zircaloy-4 processed by high-pressure torsion: A comparative study 2012, 310-313.

https://ro.uow.edu.au/engpapers/4878 


\section{Authors}

Zhiyang Wang, Huijun Li, Ulf Garbe, Mark D. Callaghan, Yanbo Wang, and Xiaozhou Liao 


\section{Microstructural evolution during gaseous hydrogen charging of Zircaloy-4 processed by}

\section{high-pressure torsion: A comparative study}

Zhiyang Wang ${ }^{\text {a,b, }}$, Huijun Li ${ }^{\text {a }}$, Ulf Garbe ${ }^{\mathrm{b}}$, Mark D. Callaghan ${ }^{\mathrm{a}}$, Yanbo Wang ${ }^{\mathrm{c}}$ and Xiaozhou Liao $^{c}$

${ }^{\text {a }}$ Faculty of Engineering, University of Wollongong, Northfields Avenue, Wollongong, NSW 2522, Australia

${ }^{\mathrm{b}}$ Australian Nuclear Science and Technology Organisation, The Bragg Institute, New Illawarra Road, Lucas Heights, NSW 2234, Australia

${ }^{c}$ School of Aerospace, Mechanical and Mechatronic Engineering, The University of Sydney, NSW 2006, Australia

\section{Abstract}

The original and high-pressure torsion (HPT) processed Zircaloy-4 materials were hydrided using gaseous hydrogen charging at different hydrogen pressures (10,15 and $20 \mathrm{~atm})$. The phase and microstructural evolutions of the samples during hydriding were characterized. It showed that when hydriding at the identical conditions, more hydrides tended to form in the HPT samples compared to that of the original ones. At a hydrogen pressure of 20 atm, the HPT sample was completely converted to $\varepsilon-\mathrm{ZrH}_{2}$ while some $\delta-\mathrm{ZrH}_{1.66}$ hydrides (volume fraction $\sim 5.73 \%$ ) were present in the material without HPT preprocessing. The HPT samples exhibited high potential for the hydride precipitation, and the large concentration of lattice defects induced by HPT was considered to be responsible for this enhanced susceptibility.

Keywords: Microstructure; Nuclear materials; Zircaloy-4; High-pressure torsion; Hydride

\section{Introduction}

* Corresponding author. Tel.: +61 2 42214143; fax: +61 2 42213238. E-mail address: zw603@uowmail.edu.au 
Zircaloy-4 (Zr-4) is extensively used as a structural material in nuclear reactors due to its attractive combination of low neutron absorption, good corrosion resistance and mechanical performance. However, formation of brittle hydrides in Zr-4 during service will degrade the mechanical properties significantly, through hydride embrittlement of this material. Detrimental effects are strongly dependent on the amount, distribution and morphology of hydrides formed. Hydride precipitation is an intricate process, controlled by several factors such as microstructure of $\mathrm{Zr}-4$, the hydriding conditions, and the hydrogen contents, which have appreciable influences on the final morphology of hydrides [1]. Currently, investigations on the hydride precipitation behavior in Zr-4 have attracted considerable attention.

Previous research efforts have successfully developed the bulk Zr-4 material with nanostructure and improved strength via a severe plastic deformation process - high-pressure torsion (HPT) [2]. This development led to the question if such HPT-processed Zr-4 could have enhanced properties with respect to nuclear reactor environments. Especially, the hydride embrittlement effect on the HPT-processed Zr-4 should be carefully evaluated before considering the practical application of this material in reactor. In the current letter, original and HPT-produced Zr-4 samples were gaseously hydrided using different hydrogen pressures. The phase constituent and microstructure of all samples were determined. The hydride precipitation behaviors (hydride phase evolution, morphology and concentration) in the original and HPT samples were compared, aiming to reveal the influence of HPT preprocessing on the hydride formation.

\section{Experimental}

The Zr-4 disk samples (diameter $10 \mathrm{~mm}$ and thickness $0.8 \mathrm{~mm}$ ) were used in this study. Some disks were processed using HPT for 5 resolutions. Details concerning the description of the HPT processing and the characterization of the HPT-induced microstructural evolution could be found in 
Ref. [2]. The as-received and HPT-processed samples were hydrogen-charged in a vacuum furnace using high purity hydrogen (99.9\%) at hydrogen pressures of 10, 15 and $20 \mathrm{~atm}$. A thermal cycling process was implemented in which the samples were soaked for $5 \mathrm{hrs}$ at a temperature of $450{ }^{\circ} \mathrm{C}$, followed by furnace cooling with a cooling rate of $\sim 2.2^{\circ} \mathrm{C} / \mathrm{min}$. Three thermal cycles were applied for all hydrided samples.

Phases in the un-hydrided and hydrided Zr-4 samples were identified by a GBC MMA X-ray diffractometer (XRD). The hydride volume fractions and hydrogen content ([H]) achieved for each hydrided sample were estimated based on the integrated intensities of specific XRD peaks using the calculation method described in [3]. Samples for metallurgical examinations were prepared according to standard procedures, and etched with a solution of $10 \mathrm{~mL} \mathrm{HF,} 100 \mathrm{~mL} \mathrm{HNO}_{3}$ and 100 $\mathrm{mL}$ distilled water for $30 \mathrm{~s}$. The microstructural characterization was performed using a JEOL JSM-6490LA scanning electron microscope (SEM).

\section{Results and discussion}
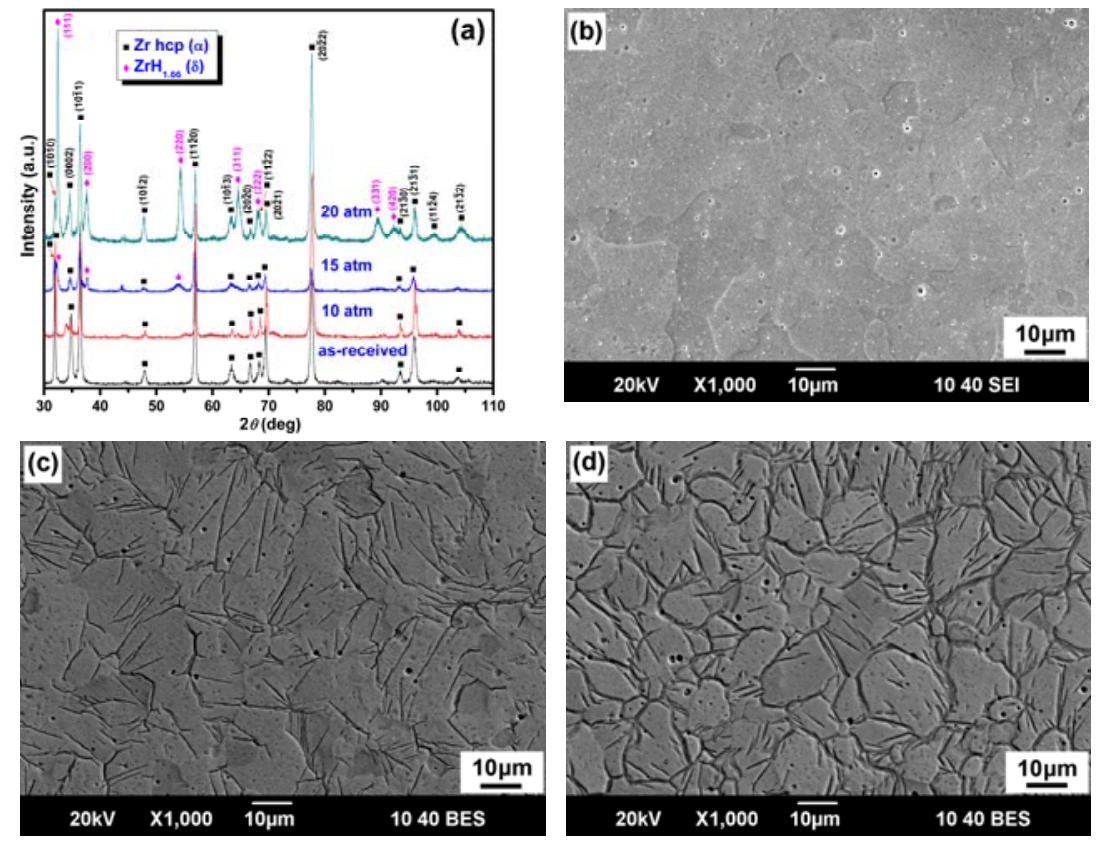

Fig. 1 shows the phase and microstructure evolutions of the as-received $\mathrm{Zr}-4$ disks before and after hydrogen charging at different hydrogen pressures. A single hexagonal-close-packed (hcp) 
$\alpha-\mathrm{Zr}$ was clearly detected in the as-received sample, and face-centred-cubic (fcc) $\delta-\mathrm{ZrH}_{1.66}$ and $\alpha-\mathrm{Zr}$ were identified in the hydrided samples at hydrogen pressures of 15 and 20 atm, whereas no distinct hydride peaks were observed in the 10 atm hydrided sample (Fig. 1a). Moreover, the XRD semi-quantification evaluation on the hydride contents and hydrogen concentrations suggested that as the hydriding pressure rose from 15 to $20 \mathrm{~atm}$, the $\delta$-hydride volume fraction increased from $2.19 \%$ to $5.73 \%$ ([H] increased from 343 to $902 \mathrm{ppm})$. Combined with the SEM microstructural characterization on the cross-sections of hydrided samples (Figs. 1b-d), we could find that the formation of hydrides and the hydride morphology and concentration were closely related to the applied hydrogen pressure. At a relatively low hydrogen pressure of $10 \mathrm{~atm}$, no hydrides were observed within the $\alpha$-Zr matrix (Fig. 1b), verifying the XRD identification (Fig. 1a). As the hydrogen pressure increased to 15 atm, needle-like hydrides appeared with most hydride needles connected with the adjacent ones (Fig. 1c). With further increasing the hydrogen pressure to 20 atm, the hydride platelets became thicker and, meanwhile, a hydride network was developed, exhibiting a higher hydride concentration (Fig. 1d).
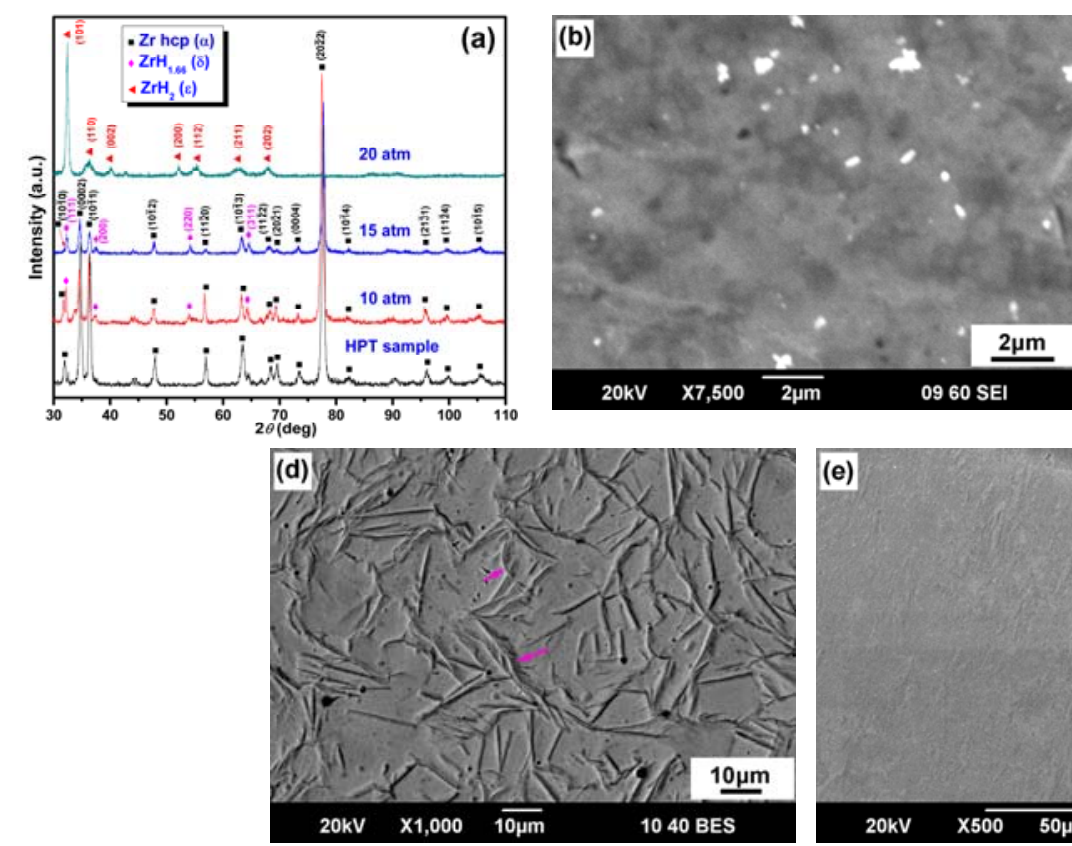

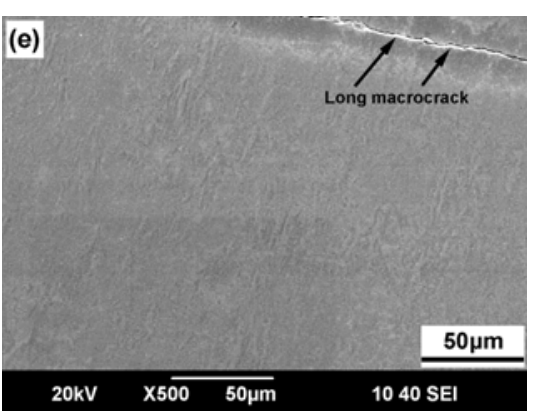


Fig. 2a shows the XRD patterns of HPT samples before and after hydriding under various hydrogen pressures. It is evident that after HPT processing the sample maintained a single $\alpha-\mathrm{Zr}$, and the $\delta-\mathrm{ZrH}_{1.66}$ emerged after hydriding the HPT samples at hydrogen pressures of 10 and $15 \mathrm{~atm}$. The XRD estimation of the $\delta$-hydride volume fractions showed that the elevation of hydriding pressures from 10 to $15 \mathrm{~atm}$ resulted in the increase of the hydride volume fractions from $2.57 \%$ to $16.20 \%$ ([H] increased from 403 to 2585 ppm). Generally, a greater concentration of hydrides could be achieved at a higher hydriding pressure for both the as-received and HPT samples. This trend may be explained by the higher hydrogen absorption of the material at the elevated hydriding pressure, according to the Sievert's law [4] which is expressed as: $C_{\mathrm{H}}=k \cdot p_{\mathrm{H}_{2}}{ }^{1 / 2}$, where $C_{\mathrm{H}}$ is the atomic percentage of hydrogen dissolved in Zircaloy, $k$ is the Sievert's constant, and $p_{\mathrm{H}_{2}}$ is the hydrogen partial pressure. When the concentration for the absorbed hydrogen exceeds the terminal solid solubility (TSS), hydrides will precipitate and, therefore, the greater hydrogen absorption at a higher hydrogen pressure will lead to the formation of a larger amount of hydrides. Interestingly, on further increasing the hydrogen pressure to $20 \mathrm{~atm}$, the hydrided sample was fully indexed to the face-centred-tetragonal (fct) $\varepsilon-\mathrm{ZrH}_{2}$, implying the formation of pure $\varepsilon$-hydride at an even higher hydrogen content $\left(\sim 21,600 \mathrm{ppm}\right.$ for pure $\left.\varepsilon-\mathrm{ZrH}_{2}\right)$. The XRD results showed that $\delta$-hydride transformed to $\varepsilon$-hydride when the hydrogen content reached a certain level, and further investigation is required to clarify the exact role of hydrogen content on the phase evolution of hydrides.

The microstructural changes of the HPT samples before and after hydriding are illustrated in Figs. 2b-e. As shown in Fig. 2b, the HPT sample exhibited a very fine grain structure which cannot be clearly revealed using SEM. Nevertheless, the previous observation using transmission electron microscopy showed that the HPT sample displayed a uniform nanocrystalline structure [2]. At a 
hydrogen pressure of $10 \mathrm{~atm}$, the microstructure of the hydrided sample essentially consisted of $\alpha-Z r$ grains and needle-shaped hydrides (Fig. 2c). The characteristic hydride morphologies exhibited a distinct variation with the hydrogen pressure change. When the hydrogen pressure increased to $15 \mathrm{~atm}$, the hydride platelets became thicker, and the hydride concentrations increased markedly; some long hydrides possessing tangled short-platelet branches were also observed (Fig. 2d). At higher hydrogen pressure of 20 atm, the platelet-morphology hydride identified as $\delta-\mathrm{ZrH}_{1.66}$ developed into the pure $\varepsilon-\mathrm{ZrH}_{2}$ (Fig. 2a), and the long macrocracks were present on the microstructure of the $\varepsilon-\mathrm{ZrH}_{2}$ sample. No apparent grain structure of $\varepsilon-\mathrm{ZrH}_{2}$ was revealed (Fig. 2e), similar to $\mathrm{Xu}$ and Shi's observation on $\varepsilon-\mathrm{ZrH}_{1.83}$ [5]. The macrocracks appeared to be generated during grinding and polishing processes, indicating the brittle nature of $\varepsilon$-hydrides. The accompanied large internal stress and microcrack with the $\varepsilon$-hydride precipitation expansion may play an essential role in this macrocrack development.

A comparison of the microstructural characterization results of the hydrided samples clearly implies that more hydrides tend to form in the HPT samples than that in the as-received samples when hydrided at the same hydrogen pressures. Note that the severe plastic deformation of the HPT samples will generate a large concentration of lattice defects (e.g., vacancies and dislocations), which is believed to be responsible for the enhanced potential for the hydride formation in the HPT samples. On the one hand, these structure defects could act as hydrogen traps, creating local domains with supersaturated hydrogen in the bulk samples during hydriding [6,7]. On the other hand, the presence of large amount of defects (especially the dislocations) provides sufficient favorable nucleation sites for hydrides, enhancing the hydride nucleation [8,9]. At a low hydrogen pressure of $10 \mathrm{~atm}$, presumably the concentration of absorbed hydrogen during hydriding is below the TSS of hydrogen at the given hydriding conditions based on the evidence that no hydrides were 
formed in the as-received samples (Figs. 1a and b). However, for the HPT sample hydrided at 10 atm, the local hydrogen concentrations in the regions of the sample with a high defect density may exceed the TSS of hydrogen due to the hydrogen trapping effect by defects, thereby leading to the precipitation of some hydrides within the HPT matrix at the low hydrogen pressure (Figs. 2a and c). On further increasing the hydrogen pressures $(>10 \mathrm{~atm})$, higher hydrogen uptake is yielded, favoring the hydride formation in both the as-received and HPT samples. Furthermore, due to the combined effects of the large concentration of lattice defects induced by HPT (acting as hydrogen traps and effective hydride nucleation sites), the nucleation and subsequent growth of hydrides are facilitated in the HPT samples. Consequently, higher hydride contents were obtained in the HPT samples than that in the as-received samples when hydrided at the same conditions.

\section{Conclusions}

A comparative study was conducted to investigate the effect of HPT preprocessing on the hydride precipitation behavior in Zr-4 during gaseous hydrogen charging. Overall, when hydriding at the same hydrogen pressures, more hydrides tended to precipitate in the HPT samples compared to that of the samples without HPT preprocessing. The HPT samples exhibited the enhanced potential for the hydride formation. This may result from comprehensive effects of a large concentration of defects induced by HPT (acting as hydrogen traps and providing preferred hydride nucleation sites), promoting the nucleation and subsequent growth of hydrides in the HPT samples.

\section{Acknowledgements}

One of the authors (Zhiyang Wang) is very grateful for the financial support of the Chinese Scholarship Council.

\section{References}

[1] Arsene S, Bai JB, Bompard P. Metall Mater Trans A 2003;34A:579-88. 
[2] Wang YB, Louie M, Cao Y, Liao XZ, Li HJ, Ringer SP, Zhu YT. Scripta Mater 2010;62:214-7.

[3] Daum RS, Chu YS, Motta AT. J Nucl Mater 2009;392:453-63.

[4] Huang JH, Huang SP. Mater Sci Eng A 1993;161:247-53.

[5] Xu JJ, Shi SQ. J Nucl Mater 2004;327:165-70.

[6] Gerard N. J Less Common Met 1987;131:13-23.

[7] Une K, Ishimoto S. J Nucl Mater 2003;322:66-72.

[8] Perovic V, Weatherly GC. J Nucl Mater 1984;126:160-9.

[9] Roy C. Atomic Energy of Canada Limited Report, AECL-2297, 1965.

\section{Figure captions}

Fig. 1. (a) XRD patterns of the as-received and corresponding hydrided samples under different hydrogen pressures; and SEM micrographs showing typical microstructures on the cross-sections of hydrided samples without HPT preprocessing under different hydrogen pressures: (b) 10; (c) 15 and (d) $20 \mathrm{~atm}$.

Fig. 2. (a) XRD patterns of the un-hydrided and hydrided HPT samples at various hydrogen pressures; and SEM images showing characteristic microstructure of (b) the HPT sample and morphologies of hydrides formed in hydrided HPT samples at hydrogen pressures of (c) 10; (d) 15 and (e) $20 \mathrm{~atm}$. 